\title{
Lang wirksame ICS/LABA-Fixkombination bessert symptomatisches Asthma
}

\author{
Bei Patienten mit symptomatischem Asthma bron- \\ chiale unter inhalativer Erhaltungstherapie verbessert \\ die Umstellung auf Fluticasonfuroat/Vilanterol die \\ Asthmakontrolle im Vergleich zur Optimierung der \\ bisherigen Therapie, so das Ergebnis einer Alltags- \\ Wirksamkeitsstudie.
}

Randomisierte kontrollierte Studien beweisen die Wirksamkeit einer therapeutischen Maßnahme an einem wohl definierten Patientenkollektiv. Die Alltagswirksamkeit bei typischen, nicht selektierten Patienten wird hingegen besser durch offene Effektivitätsstudien abgebildet, die keine nennenswerten Ein- oder Ausschlusskriterien aufweisen.

\section{"Salford Lung Study" bei Asthma bronchiale}

Eine solche pragmatische „effectiveness“-Studie ist die „Salford Lung Study Asthma“, deren Ergebnisse beim ERS vorgestellt und zeitgleich in „The Lancet“ publiziert wurden. Studienkollektiv waren 4.233 Patienten, bei denen der Hausarzt die Diagnose Asthma gestellt hatte und die dauerhaft mit inhalativen „Controller"-Medikamenten behandelt wurden.

Bei diesen Patienten wurde entweder die bisherige antiasthmatische Therapie fortgeführt bzw. wenn notwendig optimiert. Oder aber sie wurden umgestellt auf die einmal tägliche Inhalation der Fixkombination Fluticasonfuroat/Vilanterol (FF/VI, Relvar $^{\circledast}$ von GSK). Bei $78 \%$ der Studienpatienten war das Asthma nicht gut kontrolliert, der Score des Asthma-Kontrolltests lag bei unter 20 Punkten.

Diese 3.024 Patienten mit nicht ausreichender Asthmakontrolle wurden in die primäre Wirksamkeitsanalyse einbezogen. Denn der primäre Endpunkt lautete: Verbesserung des ACTScores auf Werte über 20 oder mindestens um drei Punkte.

\section{Signifikant bessere Kontrolle der Asthma-Symptomatik}

Nach einem Jahr hatten $71 \%$ der Patienten der FF/VI-Gruppe im Sinne des primären Endpunktes auf die Therapie angesprochen, im Vergleich zu 56\% in der Kontrollgruppe. Die Wahrscheinlichkeit eines Ansprechens lag unter FF/VI signifikant höher ( $\mathrm{p}<0,001)$.

FF/VI gewann den Vergleich mit der Kontrollgruppe unabhängig davon, ob die Patienten der Kontrollgruppe mit inhalativen Steroiden (ICS) allein oder mit einer Kombination aus ICS/ LABA behandelt worden sind, berichtete Studienautor Prof. Ashley Woodcock, University Hospital South Manchester, beim ERS-Kongress in Mailand. Die Häufigkeit von Nebenwirkungen war in beiden Gruppen gleich. Pneumonien waren selten. Exazerbationen traten in beiden Gruppen mit gleicher Häufigkeit auf.

Wie kam es, dass FF/VI die Asthmakontrolle über den gesamten Studienzeitraum gegenüber einer Therapie mit ICS oder anderen ICS/LABA-Kombinationen verbesserte? Die Autoren verweisen darauf, dass eine Stärke der nicht randomisierten Praxisstudie sei, die Vorteile etwa einer Einmalgabe am Tag oder eines besonders guten Inhalations-Devices herauszuarbeiten. Andererseits sei der Asthma-Control-Test-Score ein relativ schwacher Endpunkt. Es sei nicht auszuschließen, dass zu den Vorteilen in diesem Endpunkt auch die Tatsache beigetragen habe, dass dem Patient gesagt wurde, er bekäme statt seiner bisherigen Therapie nun etwas, was neu und besonders gut sei.

Dr. med. Dirk Einecke

Quellen: European Respiratory Society Congress 2017, Mailand, 9.-13.9.2017; Woodcock A, et al. Effectiveness of fluticasone furoate plus vilanterol on asthma control in clinical practice: an open-label, parallel group, randomized controlled trial. The Lancet 2017, doi:10.1016/S0140-6736(17)32398-X

\section{Tiotropium-Therapie im COPD-Frühstadium in Studie getestet}

In einer Studie mit Patienten in frühen COPD-Stadien verbesserte Tiotropium die Lungenfunktion sowie den FEV $\mathrm{V}_{1}$-Abfall nach Bronchodilatator-Applikation über zwei Jahre. Doch die Frage, ob die Frühtherapie den Krankheitsverlauf günstig beeinflusst, beantwortet die Studie nicht überzeugend.

Patienten in den COPD-Frühstadien erhalten aufgrund der Symptomarmut häufig keine Medikation. Auf der anderen Seite ist bekannt, dass der Verlust der Lungenfunktion gerade in den frühen Stadien am stärksten ist. Im COPD-Stadium 1 emp- fehlen die Leitlinien pharmakologisch eine Bedarfstherapie mit kurzwirksamen Bronchodilatatoren. Im Stadium 2 wird bereits ein LAMA oder ein LABA empfohlen.

Insofern weist die chinesische Doppelblindstudie von Y. Zhou et al. eine gewisse Schwäche auf: Sie rekrutierte zwar 841 Patienten in frühen COPD-Stadien, davon über $70 \%$ mit einem CAT-Score unter 10 und 44\% im Stadium 1. Die Mehrzahl der Studienpatienten waren jedoch im Stadium 2, für die Tiotropium eine leitliniengerechte Therapie darstellt.

Die Patienten wurden zwei Jahre lang entweder mit Tiotropium (Spiriva $18 \mu \mathrm{g} / \mathrm{d}$ ) oder Placebo behandelt. Primärer Endpunkt war der $\mathrm{FEV}_{1}$-Unterschied zwischen den Gruppen vor der 